\title{
Cell-Free miR-27a, a Potential Diagnostic and Prognostic Biomarker for Gastric Cancer
}

Jong-Lyul Park ${ }^{1}$, Mirang Kim ${ }^{1,2}$, Kyu-Sang Song ${ }^{3}$, Seon-Young Kim ${ }^{1,2}$, Yong Sung Kim ${ }^{1,2}$

${ }^{1}$ Epigenome Research Center, Genome Institute, KRIBB, Daejeon 34141, Korea, ${ }^{2}$ Department of Functional Genomics, Korea University of Science and Technology, Daejeon 34141, Korea, ${ }^{3}$ Department of Pathology, Chungnam National University College of Medicine, Daejeon 35015, Korea

http://dx.doi.org/10.5808/GI.2015.13.3.70

Genomics Inform 2013;13(3):70-75

The funding acknowledgment in this article was partially omitted as published. Additional acknowledgment is as follows:

This work was supported by the National Research Foundation of Korea (NRF), grants funded by the Korea government (MSIP; No. 2011-0030049 and 2013R1A1A2006621), and a KRIBB Research Initiative Grant. 